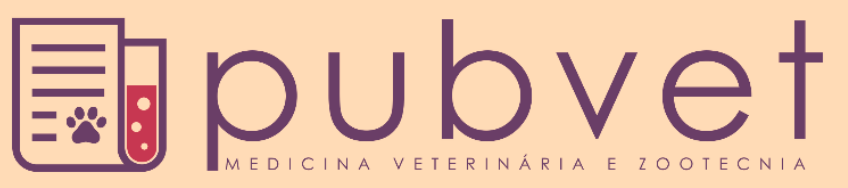

https://doi.org/10.31533/pubvet.v15n12a976.1-6

\title{
Avaliação in vitro de formulação homeopática no controle do carrapato bovino Rhipicephalus (Boophilus) microplus
}

\author{
Vanessa Cristina Dalprai Paz ${ }^{* \bullet} \mathbb{D}$, Cleiton José Paz $^{2} \bullet$, Sérgio Cangassú Santana² \\ ${ }^{I}$ Mestre em Saúde, Bem-Estar Animal e Produção Animal Sustentável. Universidade Federal da Fronteira Sul, Chapecó - SC \\ ${ }^{2}$ Pesquisadores da Empresa Hágil Terapêutica Ltda. Teófilo Otoni, Minas Gerais, Brasil \\ *Autor para correspondência, E-mail: vanessa_vcd@hotmail.com
}

\begin{abstract}
Resumo. A homeopatia veterinária está sendo cada dia mais reconhecido e difundido por ser uma alternativa que causa menos danos ao meio ambiente e aos animais, sendo principalmente, utilizada no controle de ectoparasitas. Um dos principais ectoparasitas que causam grande prejuízo econômico a pecuária brasileira é o carrapato-bovino, Rhipicephalus (Boophilus) microplus. No intuito de contribuir para aplicação de uma terapia alternativa para o controle de ectoparasito R.(B.) microplus em bovinos, a presente pesquisa teve como objetivo, avaliar a oviposição e a taxa de eclosão em teleóginas coletadas em uma propriedade experimental tratada com medicamento homeopático, bem como seus demais parâmetros biológicos. No experimento in vitro, as teleóginas foram coletadas, lavadas e fixadas em decúbito dorsal em placas de Petri, levadas a uma câmara de germinação por 12 dias em $27^{\circ} \mathrm{C} \pm 1$ e umidade relativa de $80 \%$, para obtenção de posturas, após foram pesadas as alíquotas de ovos, adicionados em tubos de Falcon e mantidos na câmara de germinação por 14 dias com temperatura de em $27^{\circ} \mathrm{C} \pm 1$ e de $80 \%$, verificando a porcentagem de eclosão. Observou-se que a formulação homeopática auxilia no tratamento e controle de infestações de $R$.(B.) microplus em bovinos criados em propriedade com histórico de resistência parasitária.
\end{abstract}

Palavras-chave: Homeopatia veterinária, parâmetros biológicos

\section{In vitro evaluation of homeopathic formulation in the control of bovine tick Rhipicephalus (Boophilus) microplus}

\begin{abstract}
Veterinary homeopathy is being increasingly recognized and spread because it is an alternative that causes less damage to the environment and to the animals, being mainly used in the control of ectoparasites. One of the main ectoparasites that cause great economic damage to Brazilian livestock is the cattle tick, Rhipicephalus (Boophilus) microplus. In order to contribute to the application of an alternative therapy for the control of the ectoparasite $R$. (B.) microplus in cattle, the present research aimed at evaluating the oviposition and hatching rate in teleogines collected from an experimental property treated with homeopathic medicament, as well as its other biological parameters. In the in vitro experiment, the teleogines were collected, washed and fixed in dorsal decubitus in petri dishes, taken to a germination chamber for 12 days at $27^{\circ} \mathrm{C} \pm 1$ and relative humidity $80 \%$, to obtain postures, after which aliquots of eggs were weighed, added to Falcon tubes and kept in the germination chamber for 14 days at a temperature of $27^{\circ} \mathrm{C} \pm 1$ and relative humidity $80 \%$, verifying the hatching percentage. It was observed that the homeopathic formulation aids in the treatment and control of $R$.(B.) microplus infestations in cattle raised on a property with a history of parasite resistance.
\end{abstract}

Keywords: Veterinary homeopathy, biological parameters 


\section{Introdução}

O carrapato bovino Rhipicephalus (Boophilus) microplus (Acari: Ixodidae) é um dos ectoparasitas mais importantes para a pecuária brasileira, causando grandes prejuízos, como a redução da qualidade do couro devido às cicatrizes ocasionadas na pele do hospedeiro durante a sua alimentação; compromete a produção de carne e leite devido às doenças provocadas; bem como, transmissão de agentes infecciosos como a Anaplasma sp. e Babesia sp., que são responsáveis pelo complexo Tristeza Parasitaria Bovina (Almeida et al., 2006; Marini et al., 2010)

O controle dos R.(B.) microplus é realizado na grande maioria por aplicações constantes de medicamentos alopáticos, ocasionando descarte indiscriminado da solução residual de carrapaticida no ambiente, resultando no aumento da resistência dos carrapatos aos pesticidas utilizados, intoxicação dos animais e dos aplicadores, bem como a contaminação de solos e águas (Gomes, 1998; Silveira et al., 2014).

A homeopatia veterinária é um método alternativo utilizado no tratamento e controle de diversas enfermidades e distúrbios orgânicos em animais, como os ectoparasitas, dentre eles o carrapato bovino (Souza, 2002). A terapia homeopática em rebanhos brasileiros está em grande expansão, por ser uma alternativa composta por produtos de origem animal, livres de resíduos contaminantes, sendo utilizada no tratamento e controle de infestações por artrópodes, verminoses, mastites, dermatites, intoxicações, distúrbios reprodutivos, imunodeficiências, entre outras.

No intuito de contribuir para aplicação de uma terapia alternativa e de comprovar que o medicamento homeopático realiza o controle de ectoparasito $R$. (B.) microplus em bovinos, a presente pesquisa teve como objetivo, avaliar a oviposição e a taxa de eclosão em teleóginas coletadas em uma propriedade experimental tratada com medicamento homeopático, bem como seus demais parâmetros biológicos.

\section{Material e métodos}

\section{Protocolo experimental}

Para a formação do experimento in vitro, foi coletado carrapatos $R$. (B.) microplus com tamanho de 4,5 a 8mm (Figura 1), de vinte vacas adultas, raça Holandesa e Jersey, em diferentes estágios de lactação, de uma propriedade de agricultura familiar da cidade de Pérola do Oeste, Paraná, Brasil. Essas vacas foram distribuídas em grupo controle $(n=10)$ e grupo tratado $(n=10)$. O medicamento homeopático para análise foi fornecido ao grupo tratado em conjunto com a alimentação no cocho, na dose de $10 \mathrm{~g} / \mathrm{animal} / \mathrm{dia}$, via oral, durante 12 meses, após este período, foi realizada a coleta das teleóginas (fêmeas ingurgitadas) de R.(B.) microplus para o experimento in vitro.

Sendo utilizada a formulação do medicamento homeopático em pó Endecthon ${ }^{\circledR}$ (da empresa Hágil Terapêutica Ltda.) composta por Ascaridinum $12 \mathrm{CH}$, Berne $9 \mathrm{CH}$, Carrapato bovino $12 \mathrm{CH}$, Carrapato do campo $12 \mathrm{CH}$, Carrapato equino 19CH, Cina 9CH, Cisticerco 30CH, Jalapa 6CH, Mosca dos chifres $12 \mathrm{CH}$, Mosca doméstica 12CH, Oxiurus 12CH, Staphisagria 12CH, Silicea 12CH, Strongyiloides $30 \mathrm{CH}$, Sulfur $12 \mathrm{CH}$, Taenia saginata $21 \mathrm{CH}$, Bixa orelanna, Sacarose q.s.p. $400 \mathrm{~g}$., na dinâmica populacional de carrapatos $R$. (B.) microplus em bovinos.

A avaliação do experimento in vitro foi adaptada dos trabalhos de Andreotti et al. (2016). Para a montagem do experimento in vitro ocorreram primeiramente, as coletas das teleóginas no início da manhã, após foram lavadas, secadas, pesadas e fixadas em decúbito dorsal em placas de Petri; divididas em grupos controle e tratadas, sendo dispostas 10 teleóginas por placa, totalizando 5 placas por grupo, gerando um total de 50 teleóginas (Figura 2).

Por fim, foi observada a porcentagem de eclosão dessas massas de ovos, no qual, foi realizado por três profissionais da área da veterinária, sendo assim, possível uma média para determinar qual grupo (controle e tratadas) obtiveram maior eclosão.

No segundo momento, as placas de Petri foram levadas em uma câmara de germinação por 12 dias em $27^{\circ} \mathrm{C} \pm 1$ e umidade relativa de $80 \%$, para obtenção de posturas (Figura 3). Após este período foram pesadas as massas dos ovos obtidos, adicionados em tubos de Falcon, com capacidade de $15 \mathrm{~mL}$ e tampadas com algodão para oxigenação, sendo mantidos na câmara de germinação por 14 dias com temperatura de $27^{\circ} \mathrm{C} \pm 1$ e umidade relativa de $80 \%$, para verificação da eclosão das ninfas (Figura 4). 


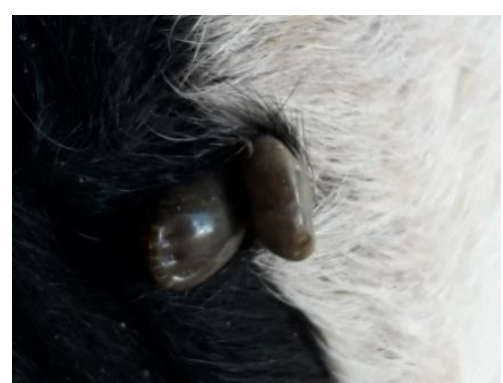

Figura 1. Carrapato $R$. (B.) microplus infestado naturalmente em vacas adultas.

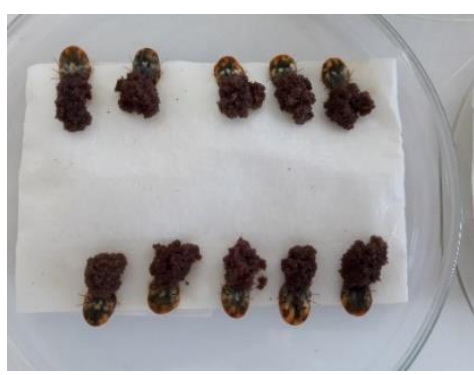

Figura 3. Teste de oviposição das teleóginas.

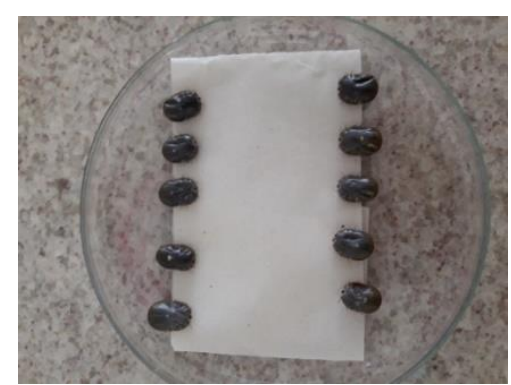

Figura 2. Montagem do experimento in vitro.

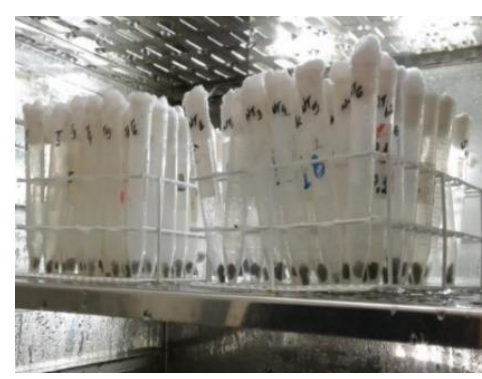

Figura 4. Teste de eclosão das ninfas.

\section{Resultados e discussão}

\section{Experimento in vitro}

A média do peso das teleóginas (Gráfico 1) que no grupo controle foi de $0,249 \mathrm{~g}$ e do grupo tratado foi de $0,227 \mathrm{~g}$. A média do massa dos ovos (Gráfico 2) que no grupo controle foi de $0,130 \mathrm{~g}$ e do grupo tratado foi de $0,120 \mathrm{~g}$, bem como a média de peso das quenóginas (fêmea ao final de sua postura) que no grupo controle foi de $0,119 \mathrm{~g}$ e do grupo tratado foi de $0,107 \mathrm{~g}$ (Gráfico 3), podemos analisar que as teleóginas do grupo controle possui maior massa de peso com ovos, sem ovos e dos ovos, ou seja, em relação ao grupo dos tratados, podemos dizer que elas são maiores, se alimentam mais nos animais (vacas), produzem mais ovos e consequentemente, gerando maior número de ninfas e consequentemente ocasionando maior infestação.

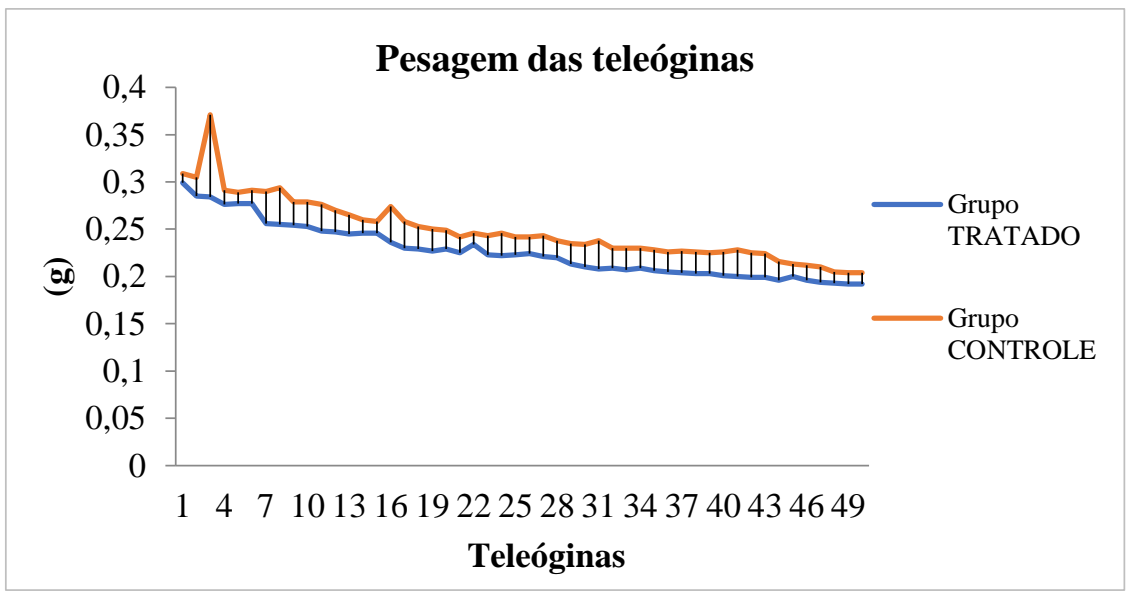

Gráfico 1. Pesagem das teleóginas dos grupos controle e tratados.

Analisando a média total da porcentagem de eclosão dos ovos dos carrapatos tratados, podemos verificar que o valor foi bem representativo (70\%), isso pode ter ocorrido devido que o medicamento homeopático tem sua funcionalidade ao longo prazo, porém, levando em consideração a média total da porcentagem de eclosão dos ovos dos carrapatos do grupo controle (86\%), podemos observar que os animais tratados com o medicamento homeopático, tem um maior controle na propagação das ninfas de carrapatos (Gráfico 4).

A partir desses dados foi avaliado o Índice de Eficácia Reprodutiva (IER), o Índice Nutricional (IN), de acordo com Bennett (1974) e a Eficiência Reprodutiva (ER) de cada grupo usando as seguintes 
fórmulas:

$$
\begin{aligned}
& I E R=\frac{\text { massa dos ovos }(g)}{\text { massa das teleóginas }(g)} \times 100 \\
& \text { I } N=\frac{\text { massa dos ovos }(g)}{\text { massa das teleóginas }(g)-\text { massa das quenóginas }(g)} \times 100 \\
& E R=\frac{\text { peso da massa de ovos }(g) \times \text { porcentagem deeclosão }}{\text { peso da fêmea }(g)}
\end{aligned}
$$

Dessa forma, obtemos os seguintes resultados, o Índice de Eficácia Reprodutiva (IER) nos carrapatos do grupo controle foi de $54 \%$ e do tratado foi de 53\%; em relação ao Índice Nutricional (IN), obtivemos $43 \%$ em ambos os grupos e a Eficiência Reprodutiva (ER) do grupo controle foi de 0,4815 e do grupo tratado foi de 0,3993 .

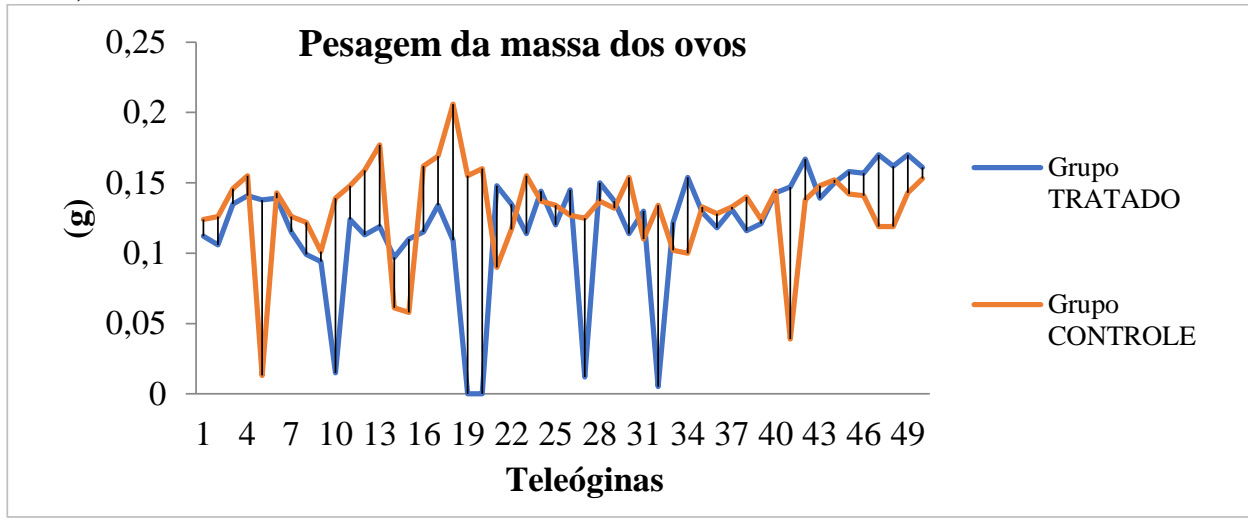

Gráfico 2. Pesagem das massas dos ovos das teleóginas dos grupos controle e tratados.

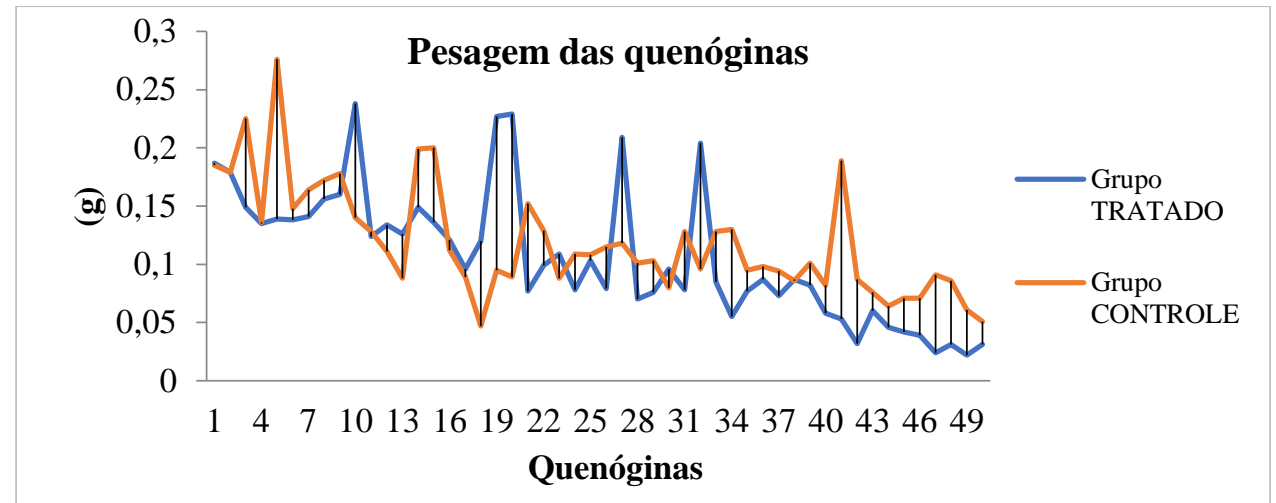

Gráfico 3. Pesagem das quenóginas dos grupos controle e tratados.

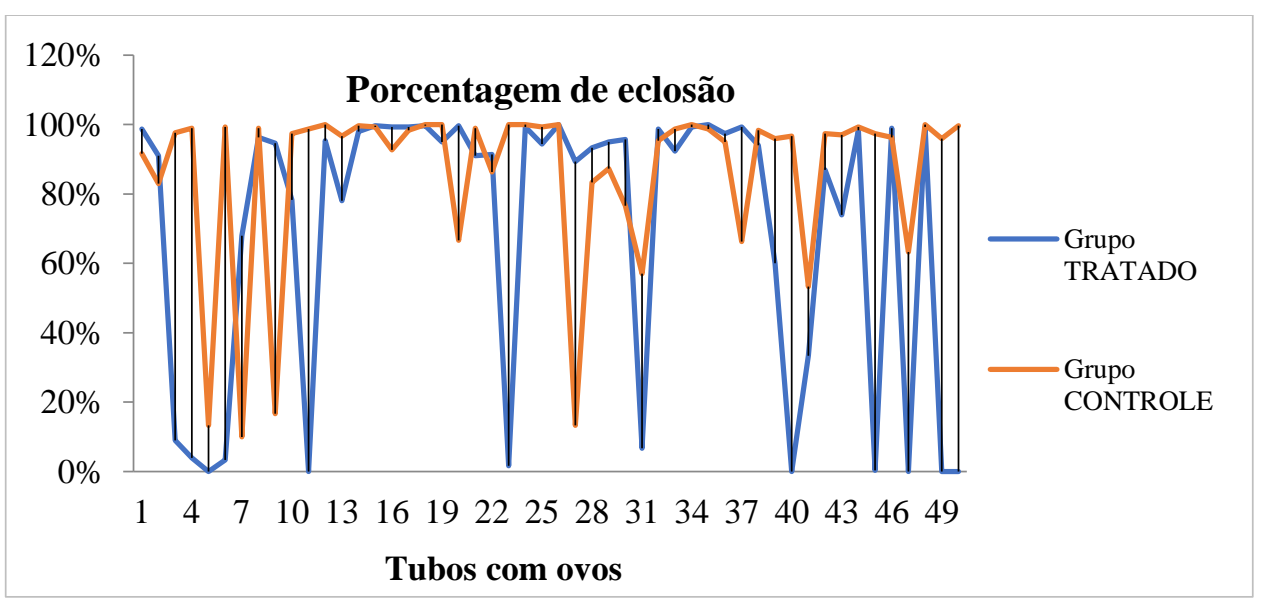

Gráfico 4. Porcentagem de eclosão dos ovos dos grupos controle e tratados.

Conforme demonstrado na Tabela 1 , não houve diferença $(\mathrm{P}>0,05)$ entre os grupos controle e o grupo tratado em relação aos parâmetros biológicos. 
As diferenças nos parâmetros reprodutivos (IER e ER) e nutricional (IN), das fêmeas ingurgitadas avaliadas in vitro no presente estudo, não foram estatisticamente significantes; porém, em relação às massas das teleóginas, dos ovos e das quenóginas, podemos observar que houve um valor significante; já em relação às taxas de eclosão, seus dados estatísticos não foram significantes.

Nos diferentes experimentos, foram obtidos resultados positivos em relação à eficácia do produto homeopático testado, mesmo que em alguns deles tenha sido de forma mais singelo, assim, ocorrendo uma redução da infestação de carrapatos nas vacas, um controle nas pastagens, diminuição da irritação e incômodo que o carrapato ocasiona nos animais, isso resultará em uma maior produção leiteira.

A ação do medicamento homeopático, nos carrapatos $R$. (B.) microplus, ocorre pelo estímulo das defesas do hospedeiro, não interferindo de modo direto na fertilidade dos carrapatos, mas, interferindo em sua alimentação e desenvolvimento, porém, possibilitando a ocorrência de oviposição com eclosão, que ao longo do tempo, o medicamento homeopático auxiliará em uma redução e proporcionará um controle desses ectoparasitos nas propriedades. Isso ocorre, devido que os estímulos energéticos do medicamento homeopático agem sobre as terminações nervosas da mucosa bucal, trato digestivo e pele, desencadeando uma sequência de impulsos nervosos em cadeia, por via reflexa, ao Sistema Nervoso Central e deste até as terminações nervosas em nível da pele, estimulando os mecanismos locais das defesas do organismo, devido que o medicamento homeopático é composto por associações de princípios ativos e agem sinergicamente no combate desses ectoparasitas (Gemelli \& Pereira, 2018).

Com relação aos resultados médios da comparação in vitro do desempenho reprodutivo das fêmeas ingurgitadas coletados de ambos os grupos e incubados em laboratório, em um experimento semelhante, Figueiredo et al. (2018) relataram que não foram observadas diferenças para esses parâmetros entre os dois grupos, assim, corroborando com a nossa pesquisa.

Tabela 1. Parâmetros biológicos de fêmeas ingurgitadas de $R$. (B.) microplus em bovinos do grupo controle e do grupo tratado com medicamento homeopático.

\begin{tabular}{lccccc}
\hline Parâmetro biológicos & \multicolumn{2}{c}{ Grupo controle } & \multicolumn{2}{c}{ Grupo tratado } & \multicolumn{2}{c}{ Valor $\mathrm{p}$} \\
& Média de massa & Desvio padrão & Média de massa & Desvio padrão & \\
\hline Massa das teleóginas (g) & 0,242 & 0,0324 & 0,2270 & 0,0280 & $<0,05$ \\
Massa de ovos (g) & 0,1355 & 0,0331 & 0,1295 & 0,0423 & $<0,05$ \\
Massa de quenóginas (g) & 0,1055 & 0,0470 & 0,0960 & 0,0561 & $<0,05$ \\
Eclodibilidade (\%) & 86 & 0,2441 & 70 & 0,3988 & $>0,05$ \\
IER & 53,719 & - & 52,863 & - & $>0,05$ \\
IN & 43,169 & - & 43,263 & - & $>0,05$ \\
ER & 0,4815 & - & 0,3993 & - & $>0,05$ \\
\hline
\end{tabular}

Gazim et al. (2010) observaram, em um ensaio in vitro, que houve uma diferença significativa na taxa de incubação dos ovos, a eficácia do bioterápico do carrapato na taxa de eclosão dos ovos foi de $50 \%$ no grupo tratado e $85-95 \%$ no grupo controle, resultando na redução do peso das fêmeas, a diminuição da massa de ovos e consequentemente, na taxa de eclosão dos ovos.

Santos et al. (2016) obtiveram resultados semelhantes à da nossa pesquisa em relação aos parâmetros biológicos, não obtendo resultados de forma significativa, que resultaram na comprovação do controle dos carrapatos com isopatia.

\section{Conclusão}

$\mathrm{O}$ experimento in vitro, pode nos mostrar diferentes resultados em relação à eficácia deste medicamento homeopático. No experimento in vitro, referente às pesagens das teleóginas coletadas nos animais, nas pesagens das massas dos ovos postos pelas teleóginas e na porcentagem de eclosão desses ovos, pode observar que, as dos animais tratados foi inferior do que ao do controle. Nos demais parâmetros, os resultados foram semelhantes entre os grupos. Dessa forma, podemos concluir que o medicamento homeopático faz o controle no processo reprodutivo e no ciclo do carrapato, diminuindo assim, a infestação nos animais e nas propriedades.

\section{Referências bibliográficas}

Almeida, M. B., Tortelli, F. P., Riet-Correa, B., Ferreira, J. L. M., Soares, M. P., Farias, N. A. R., RietCorrea, F., \& Schild, A. L. (2006). Tristeza parasitária bovina na região sul do Rio Grande do Sul: 
estudo retrospectivo de 1978-2005. Pesquisa Veterinária Brasileira, 26(4), 237-242.

Andreotti, R., Koller, W. W., \& Garcia, M. V. (2016). Carrapatos: protocolos e técnicas para estudo. In Embrapa Gado de Corte. Brasília, DF: Embrapa, 2016.

Bennett, G. F. (1974). Oviposition of Boophilus microplus (Canestrini)(Acarida: Ixodidae). I. Influence of tick size on egg production. Acarologia, 16(1), 52-61.

Figueiredo, A., Fantatto, R. R., Agnolon, I. C., Lopes, L. G., Oliveira, P. R., Mathias, M. I. C., Alves, T. C., Barioni, W., \& Chagas, A. C. S. (2018). In vivo study of a homeopathic medicine against Rhipicephalus (Boophilus) microplus in dairy cow. Revista Brasileira de Farmacognosia, 28, 207 213. https://doi.org/10.1016/j.bjp.2018.01.008.

Gazim, Z. C., Ferreira, F. B. P., Silva, A. V., Bolognese, K. C., Merlin, E., Messa, V., Jesus, R. A., Coutinho, C. A., \& Silva, L. C. M. (2010). Efficiency of tick biotherapic on the control of infestation by Rhipicephalus (Boophilus) microplus in Dutch dairy cows. International Journal of High Dilution Research, 9(33), 156-164.

Gemelli, J. L., \& Pereira, A. S. C. (2018). Princípios e utilizações da homeopatia em bovinos de corte. Uma Revisão. Revista Brasileira de Higiene e Sanidade Animal, 12(3), 327-341. https://doi.org/10.5935/1981-2965.20180032.

Gomes, A. (1998). Controle do carrapato do boi: um problema para quem cria raças européias. Embrapa Gado de Corte, 31(1), 1-8.

Marini, A., Soutello, R. V. G., Costa, R. L. D., Neves, J. H., Vacati, A. C., Barreto, T. N., Monte Júnior, S., \& Demarchi, J. J. A. A. (2010). Infestação por Rhipicephalus (Boophilus) microplus em novilhas de diferentes grupos genéticos. Boletim de Indústria Animal, 67(1), 65-71.

Santos, T. R. B., Aguiar, C. L. G., Prestes, L., Daneluz, M. O., Alves, B. F., \& Torres, M. I. T. (2016). Avaliação da eficácia de produtos isoterápicos no controle de Rhipicephalus (Boophilus) microplus. Arquivos Do Instituto Biológico, 82, 1-5.

Silveira, W. H., Carvalho, G. D., \& Peconick, A. P. (2014). Medidas de controle do carrapato Rhipicephalus microplus: uma breve revisão. PUBVET, 8(Art 1715), 1136-1282.

Souza, M. F. A. (2002). Homeopatia veterinária. Conferência Virtual Global Sobre Produção Orgânica de Bovinos de Corte, 1, 1-4.

Histórico do artigo:

Recebido: 5 de julho de 2021

Aprovado: 19 de agosto de 2021

Disponível online: 4 de novembro de 2021
Licenciamento: Este artigo é publicado na modalidade Acesso Aberto sob a licença Creative Commons Atribuição 4.0 (CC-BY 4.0), a qual permite uso irrestrito, distribuição, reprodução em qualquer meio, desde que o autor e a fonte sejam devidamente creditados. 